\title{
Astrochemistry in external galaxies: how to use molecules as probes of their physical conditions
}

\author{
Serena Viti \\ Department of Physics and Astronomy, University College London, Gower St., \\ London, WC1E 6BT, UK \\ email: serena.viti@ucl.ac.uk
}

\begin{abstract}
It is now well established that chemistry in external galaxies is rich and complex. In this review I will explore whether one can use molecular emissions to determine their physical conditions. There are several considerations to bear in mind when using molecular emission, and in particular molecular ratios, to determine the densities, temperatures and energetics of a galaxy, which I will briefly summarise here. I will then present an example of a study that uses multiple chemical and radiative transfer analyses in order to tackle the too often neglected 'degeneracies' implicit in the interpretation of molecular ratios and show that only via such analyses combined with multi-species and multi-lines high spatial resolution data one can truly make molecules into powerful diagnostics of the evolution and distribution of molecular gas.
\end{abstract}

Keywords. astrochemistry, galaxies: ISM, galaxies: nuclei, galaxies: individual (NGC 1068)

\section{Introduction}

The first detections of molecular emission in external galaxies were made in the 1970s. Since then it has become clear that chemistry in external galaxies can be complex and that, although most molecular emission is probably extended, it is certainly not all coexistent. CO emission is routinely used to trace the molecular mass of a galaxy, as it traces $\mathrm{H}_{2}$ at large scale (see review by Bolatto et al. (2013)), although one notes that the $X$-factor used in the Milky Way to convert CO integrated line intensities to $\mathrm{H}_{2}$ column densities may give misleading results if applied to other galaxies (e.g. Bell et al. 2006). However, it is the observations of molecules with a high critical density that allow us to trace the reservoir for star formation or describe the process of star formation itself, including determining the influence of newly formed stars on their environments.

The objective of this review is to establish how one can use molecular emissions of different species to explore the physical conditions of galaxies and their likely evolutionary status, as we routinely do for our own Galaxy. In other words can astrochemistry provide the tools to explore the physical conditions and energetics of external galaxies?

There are two main challenges for the astrochemist. The first challenge is that even for the closest galaxies (e.g. M82) with the most powerful interferferometer to date (ALMA), the highest spatial resolution that one can achieve is $\sim 0.2 \mathrm{pc}$ which is still larger than an individual star forming core. In fact, for most galaxies a much worse resolution is achieved, with entire star-forming regions being unresolved (e.g. for NGC 1068, at a distance of $14 \mathrm{Mpc}$, the highest spatial resolution achieved is $30 \mathrm{pc}$, Garcia-Burillo et al. 2014). Hence the telescope beam will usually encompass emissions from many types of region and the molecular emission will be an 'ensemble' of multi-phase gas where the spatial and temporal effects are diluted in the beam. This will challenge the interpretation of 
molecular line emission, especially for galaxies very different from our own. The range of physical parameters (gas densities, UV fields, cosmic ray ionization rates, dust properties, etc.) will differ across galaxies (Williams \& Viti 2013). Hence we can not necessarily use information about the Milky Way as a reliable guide to the properties of other galaxies.

The second challenge for the astrochemist is the fact that molecular spectra of nearby galaxies are now proving to be almost as rich as those of the Milky Way (Aladro et al. 2011, Aladro et al. 2013, Costagliola et al. 2015). For example, an unbiased molecular line survey in the wavelength range $1.7-2.3 \mathrm{~mm}$ towards the nuclear region of the starburst galaxy NGC 253 shows the presence of more than 20 different molecular species (Martin et al. 2006), all with relatively high abundances (see Table 6.2 in Williams \& Viti 2013). The concomitant detection of, say, $\mathrm{CO}, \mathrm{SiO}$, and $\mathrm{CH}_{3} \mathrm{OH}$ emissions in a spatially unresolved galaxy does not necessarily mean that they are emitted from the same gas: the first molecule may indicate the presence of cold diffuse clouds, the second strong shocks, and the third dense star-forming cores. Hence such chemical complexity cannot be explained by a one-component model.

In order to stand up to these challenges the interpretaation of molecular emission must take into consideration the chemical routes to the formaton and destruction of each species, as well as their sensitivity to the physical and chemical conditions of the gas.

\section{How to interpret molecular observations in external galaxies}

Molecular observations are an ideal tool to trace a wide range of densities and energetics in the interstellar medium of a galaxy, largely because of the wide range of critical densities across the different molecular species and across the transitions of the same molecule, as well as the dependendencies of chemical reactions on the energy available to the system i.e the kinetic temperature of the gas. In fact star formation as well as AGN activity are both affected by the molecular gas available to the system.

Many studies have shown that molecular ratios, such as $\mathrm{HCO}^{+} / \mathrm{HCN}$ or $\mathrm{HCN} / \mathrm{CO}$ differ across different types of galaxies expecially between AGN-dominated galaxies and starburst-dominated galaxies. However the derived abundance ratios for an individual galaxy also highly differ across studies depending on the transitions observed, the available resolution, and the method used in deriving the column densities.

The differences between molecular ratios in starburst or AGN galaxies as compared to our own Galaxy have been interpreted as anomalous at times and an indication either of high ionisation rates or of high star formation rates. On the other hand, it is likely that multiple components, each with different physical conditions, are contributing to the emission (e.g. Giant Molecular Clouds, where star formation take place, will have average number densities of the order of $10^{3} \mathrm{~cm}^{-3}$ and $\mathrm{T}=50-100 \mathrm{~K}$, while the star-forming clumps may have number densities up to $\sim 10^{7} \mathrm{~cm}^{3}$ and temperatures as low as $10 \mathrm{~K}$ ), so that a knowledge of the individual components is required to interpret such ratios. This will also explain why the use of different transitions in deriving the abundance ratios as well as different beam sizes lead to substantial differences within the same galaxy. An understanding of the chemistry behind each molecule and its dependencies on the density and temperature of the gas will lead to a more coordinated approach to understanding the nature of galaxies by using molecular line emissions.

In fact it is well known that the degeneracy between density and temperature implies that single transitions of one molecule should not be used to determine quantitatively the gas density or temperature, especially in extragalactic environments. Chemical models show that almost any density can be representative of a large range of environments; for example, a density of $\leqslant 10^{4} \mathrm{~cm}^{-3}$ may indicate an ensemble of dark/quiescent gas, 
potential site of future star foramtion; on the other hand these are also typical densities of PDRs. An average density of $10^{5} \mathrm{~cm}^{-3}$ may indicate that star forming gas is dominant, but it may also be a signature of shocked-compressed gas. Finally a high density of $\sim 10^{6} \mathrm{~cm}^{-3}$ may be typical of left over gas from episodes of starbursts or highly shocked gas driven by AGN (Krips et al. 2011; Viti et al. 2014). In Figure 1 two chemical models showing the fractional abundance of selected species as a function of cosmic ray ionization rate are plotted: the top one represent a steady-state calculation using a PDR gas-phase code (Bisbas et al. 2012) at a density of $10^{5} \mathrm{~cm}^{-3}$; the bottom figure shows the results from a time dependent gas-grain chemical model (Viti et al. 2004) which is run in two phases: the first phase follows the collapse from a diffuse gas to a density of $10^{5} \mathrm{~cm}^{-3}$. The temperature is kept constant at $10 \mathrm{~K}$ and atoms and molecules freeze on to the dust and are involved in surface reactions. The second phase follows the gas as its temperature is increased to $100 \mathrm{~K}$ due to star formation taking place, with subsequent sublimation of the icy mantles formed during the first phase. The two models are clearly very different and simulate physically different environments; and yet for the same cosmic ray ionization rate, some of the abundances are remarkably similar: take for example the $\mathrm{HCN} / \mathrm{HCO}^{+}$ at a cosmic ray 100 times that of our own Galaxy. Clearly, this ratio alone would not be able to inform the observer whether it is tracing star forming gas, AGN or PDR dominated gas without any prior information or more molecular observations.

\subsection{Why are molecular ratios 'degenerate'?}

Most molecular species have more than one route of formation and destruction. For example, let us consider a collapsing core undergoing collapse from a diffuse state, e.g. $100 \mathrm{~cm}^{-3}$, to a high density of, say, $10^{7} \mathrm{~cm}^{-3}$, under standard interstellar conditions and let us look at the chemistry of $\mathrm{HCO}^{+}$and $\mathrm{HCN}$, the two most commonly observed extragalactic species, after CO. At very low densities the following routes of formation are important:

$$
\begin{gathered}
\mathrm{H}+\mathrm{HCN}^{+} \rightarrow \mathrm{HCN}+\mathrm{H}^{+} \\
\mathrm{C}^{+}+\mathrm{H}_{2} \mathrm{O} \rightarrow \mathrm{HCO}^{+}+\mathrm{H}^{+}
\end{gathered}
$$

while at intermediate densities:

$$
\begin{aligned}
& \mathrm{N}+\mathrm{CH}_{2} \rightarrow \mathrm{HCN}+\mathrm{H} \\
& \mathrm{C}+\mathrm{NH}_{2} \rightarrow \mathrm{HCN}+\mathrm{N}
\end{aligned}
$$

and

$$
\begin{gathered}
\mathrm{H}_{3}^{+}+\mathrm{CO} \rightarrow \mathrm{HCO}^{+}+\mathrm{H}_{2} \\
\mathrm{CH}+\mathrm{O} \rightarrow \mathrm{HCO}^{+}+\mathrm{e}^{-}
\end{gathered}
$$

contribute $\sim 30 \%$ each to the formation of $\mathrm{HCN}$ and $\mathrm{HCO}^{+}$respectively. Finally, at high densities:

$$
\mathrm{NH}_{3}+\mathrm{CN} \rightarrow \mathrm{HCN}+\mathrm{NH}_{2}
$$

and Reaction 2.5, are the two dominant routes for $\mathrm{HCN}$ and $\mathrm{HCO}^{+}$respectively. 

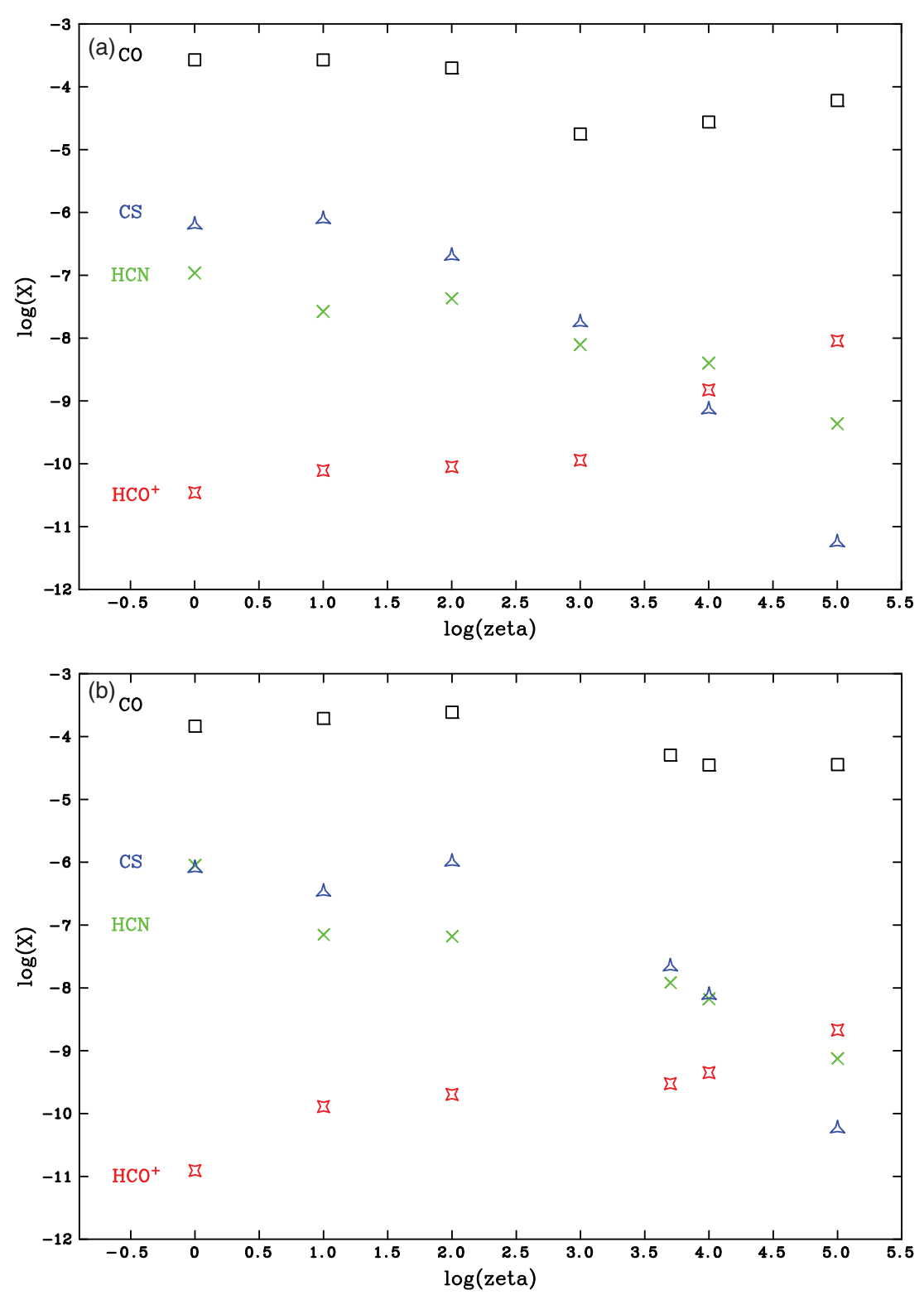

Figure 1. Fractional abundances (with respect to the total number of hydrogen nuclei) of selected species as a function of cosmic ray ionization rate for two types of chemical models. See text.

The formation and destruction routes are not just dependent on the density of course. For constant density, variation in cosmic ray ionization rate can lead to different routes of formation and destruction. For example, CS, another routinely observed molecule in extragalactic environemts, is very sensitive to cosmic ray ionization rates. At $\zeta>100 \zeta_{0}$ the main destruction mechaniscm for $\mathrm{CS}$ is: $\mathrm{CS}+\mathrm{O} \rightarrow \mathrm{CO}+\mathrm{S}$, while at low ionization rates $\mathrm{H}_{3} \mathrm{O}^{+}+\mathrm{CS} \rightarrow \mathrm{HCS}^{+}+\mathrm{H}_{2} \mathrm{O}$ is dominant.

From an observational point of view the variations in the chemistry are of course reflected in the observed line intensities. Figure 2 shows theoretical line intensities for CS for a very small grid of chemical and radiative transfer models. 

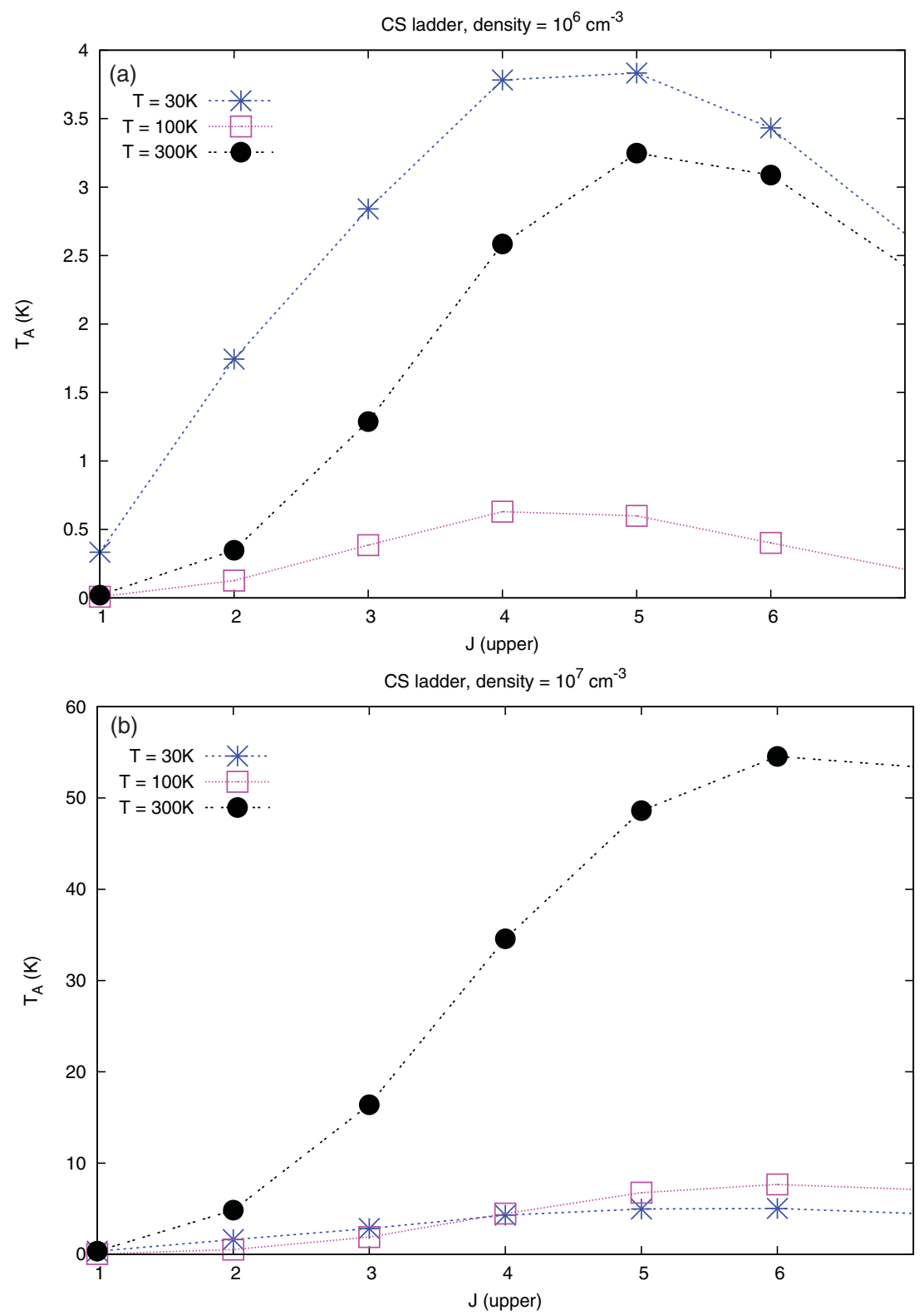

Figure 2. Theoretical CS ladders calculated by coupling chemical (UCL_CHEM, Viti et al. 2011) and line radiative transfer models (SMMOL, Kelly et al. 2015).

Already from such a small grid (where we only used two gas densities and three gas temperatures, keeping constant the cosmic ray ionisation rate and not including shocks, for example) one can see how sensitive the intensities of the CS transitions are; it is especially interesting to see how the ladder peaks at different $\mathrm{J}$ depending on the gas temperatures and densities.

When one considers that hundreds of species involved in thousands of reactions may be present in the gas, then disentangling the chemistry from the physics is not a trivial task, especially as we must not forget the role of dust and surface reactions. For example, methanol is routinely observed in extragalactic environments, and yet there is no efficient 
Table 1. Table 10 from Viti et al. (2014). Grid of chemical models. The cosmic ray ionization rate, the radiation field, the gas temperature, and the gas density are listed in columns $2-5$.

\begin{tabular}{cccccc}
\hline Model $\mathrm{n}^{o}$ & $\zeta\left(\mathrm{s}^{-1}\right)$ & $G_{0}($ Draine $)$ & $\mathrm{T}(\mathrm{K})$ & $\mathrm{n}_{\text {final/preshock }}\left(\mathrm{cm}^{-3}\right)$ & Shock \\
\hline 1 & 1 & 1 & 100 & $10^{4}$ & $\mathrm{~N}$ \\
2 & 1 & 1 & 100 & $10^{5}$ & $\mathrm{~N}$ \\
3 & 1 & 1 & 100 & $10^{6}$ & $\mathrm{~N}$ \\
4 & 10 & 1 & 100 & $10^{4}$ & $\mathrm{~N}$ \\
5 & 10 & 1 & 100 & $10^{5}$ & $\mathrm{~N}$ \\
6 & 10 & 1 & 100 & $10^{6}$ & $\mathrm{~N}$ \\
7 & 1 & 10 & 100 & $10^{4}$ & $\mathrm{~N}$ \\
8 & 1 & 10 & 100 & $10^{5}$ & $\mathrm{~N}$ \\
9 & 1 & 10 & 100 & $10^{6}$ & $\mathrm{~N}$ \\
10 & 10 & 10 & 100 & $10^{4}$ & $\mathrm{~N}$ \\
11 & 1 & 500 & 100 & $10^{4}$ & $\mathrm{~N}$ \\
12 & 500 & 1 & 100 & $10^{4}$ & $\mathrm{~N}$ \\
13 & 5000 & 1 & 100 & $10^{4}$ & $\mathrm{~N}$ \\
14 & $10^{5}$ & 1 & 100 & $10^{5}$ & $\mathrm{~N}$ \\
15 & 10 & 1 & 100 & $2 \times 10^{6}$ & $\mathrm{~N}$ \\
16 & 1 & 1 & 100 & $5 \times 10^{6}$ & $\mathrm{~N}$ \\
17 & 1 & 1 & 100 & $2 \times 10^{6}$ & $\mathrm{~N}$ \\
18 & 1 & 1 & 200 & $10^{5}$ & $\mathrm{~N}$ \\
19 & 1 & 1 & 200 & $10^{4}$ & $\mathrm{~N}$ \\
20 & 1 & 1 & 200 & $10^{6}$ & $\mathrm{~N}$ \\
21 & 10 & 1 & 200 & $10^{6}$ & $\mathrm{~N}$ \\
22 & 1 & 10 & 200 & $10^{6}$ & $\mathrm{~N}$ \\
23 & 10 & 1 & 200 & $10^{5}$ & $\mathrm{~N}$ \\
24 & 1 & 10 & 200 & $10^{5}$ & $\mathrm{Y}$ \\
25 & 1 & 1 & - & $10^{4}$ & $\mathrm{Y}$ \\
26 & 10 & 1 & - & $10^{5}$ & \\
27 & 1 & 1 & - & & \\
\hline & & & & & 5 \\
\hline
\end{tabular}

way of forming it in the gas at low temperatures $(\mathrm{T}<100 \mathrm{~K})$. It is likely that most of the $\mathrm{CH}_{3} \mathrm{OH}$ observed in extragalactic environments has formed on dust grains and then sublimated back in the gas phase.

In summary, it is important to emphasize that chemistry leads to a large range of ratios, heavily dependent on small changes in the physical and chemical (initial) conditions of the gas. Hence one cannot use molecular ratios without considering of the chemistry behind them and without paying attention to individual abundances. Performing multiple, independent analyses of the observational data is therefore essential as we shall demonstrate in the next section.

\section{NGC 1068: a case study}

The unprecedented resolution of ALMA has finally allowed us to resolve structures inside nearby galaxies. NGC 1068, a composite starburst/AGN galaxy, is a Seyfert 2 galaxy, at a distance of $\sim 14 \mathrm{Mpc}$. Many studies have focused on this galaxy (e.g. Usero et al. 2004, Israel et al. 2009, Kamenetzky et al. 2011, Hailey-Dunsheath et al. 2012, Aladro et al. 2013, Schinnerer, et al. 2000, Garcia-Burillo et al. 2010, Krips et al. 2011, Garcia-Burillo et al. 2014, Viti et al. 2014) and have shown that molecular tracers of dense gas are essential to spatially resolve the distribution, kinematics, and excitation of the circumnuclear gas of NGC 1068, as well as to study the relationship between the $r \sim 1-1.5 \mathrm{kpc}$ starburst ring and the $r \sim 200 \mathrm{pc}$ circumnuclear disc (CND) located around the AGN. In Garcia-Burillo et al. (2014) ALMA Cycle 0 observations in Bands 7 and 9 of several molecular transitions, namely CO $(3-2)$ and $(6-5), \mathrm{HCO}^{+}(4-3)$, $\mathrm{HCN}(4-3)$, and CS $(7-6)$ within the $r \sim 200$ pc CND, were presented (see Figure 3 ). 

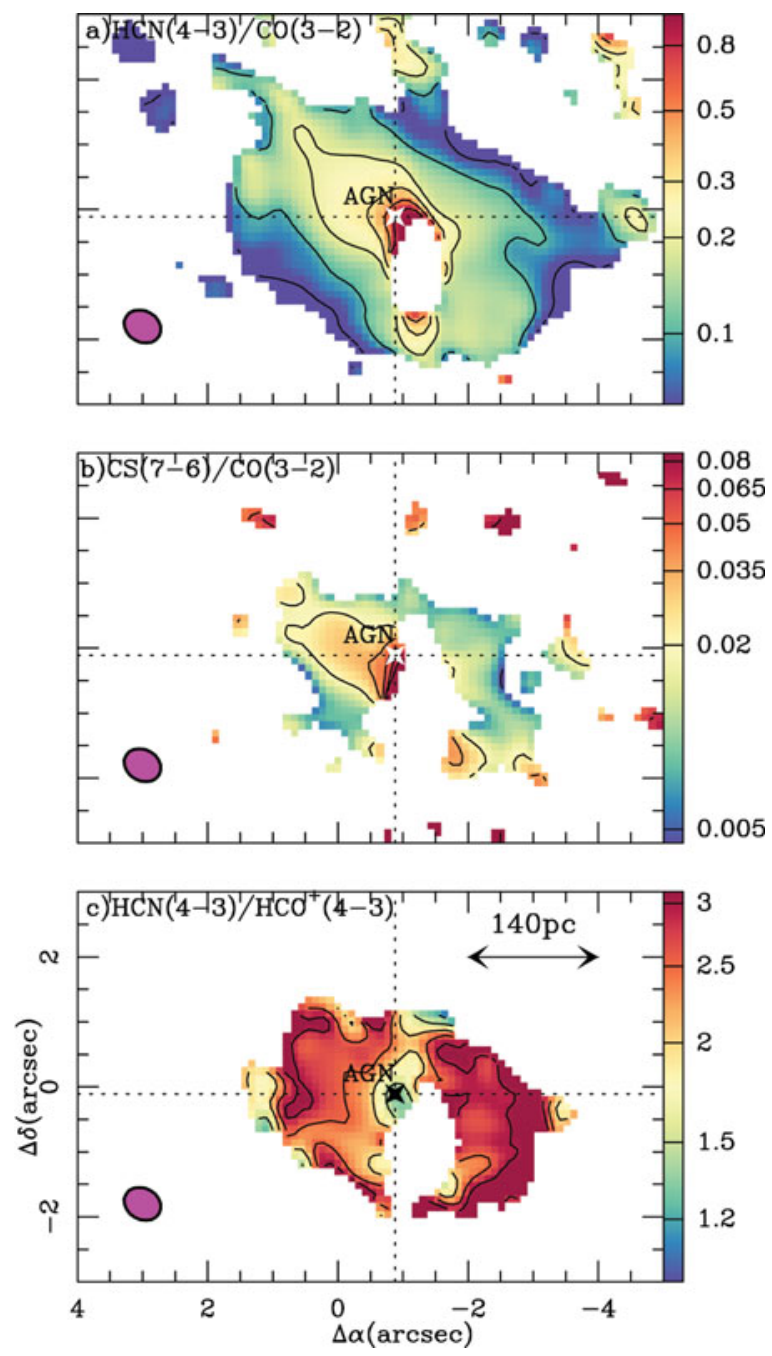

Figure 3. Figure 5 from Viti et al. (2014). $\mathrm{HCN}(4-3) / \mathrm{CO}(3-2), \operatorname{CS}(7-6) / \mathrm{CO}(3-2)$ and $\mathrm{HCN}(4-3) / \mathrm{HCO}^{+}(4-3)$ velocity-integrated intensity ratios in the CND. The filled ellipses represent the spatial resolutions used to derive the line ratio maps: $0.6^{\prime \prime} \times 0.5^{\prime \prime} \mathrm{PA}=60^{0}$, for all panels.

Viti et al. (2014) performed a chemical analysis of the gas within the CND with the aim of quantifying the chemical differentiation and of determining the chemical origin of such differentiation: the ALMA data were used together with lower resolution IRAM Plateau de Bure Interferometer data covering the lower-J transitions of each molecule. Five different analyses were performed: an LTE analysis, including a rotation diagram; three different analyses using RADEX (van der Tak et al. 2007) differing in the number of ratios and species used; and finally a purely ab initio analysis using a time dependent gas-grain chemical mode. While each analysis led to its own detailed conclusions, all five indicated that more than one gas-phase component is necessary to fit all the available molecular ratios uniquely. Chemically this is certainly not unexpected, as discussed in the section above. Of particular interest to this review is Figure 12 in Viti et al. (2014), which we include here together with their Table 10, in Figure 4 and Table 1 respectively. 


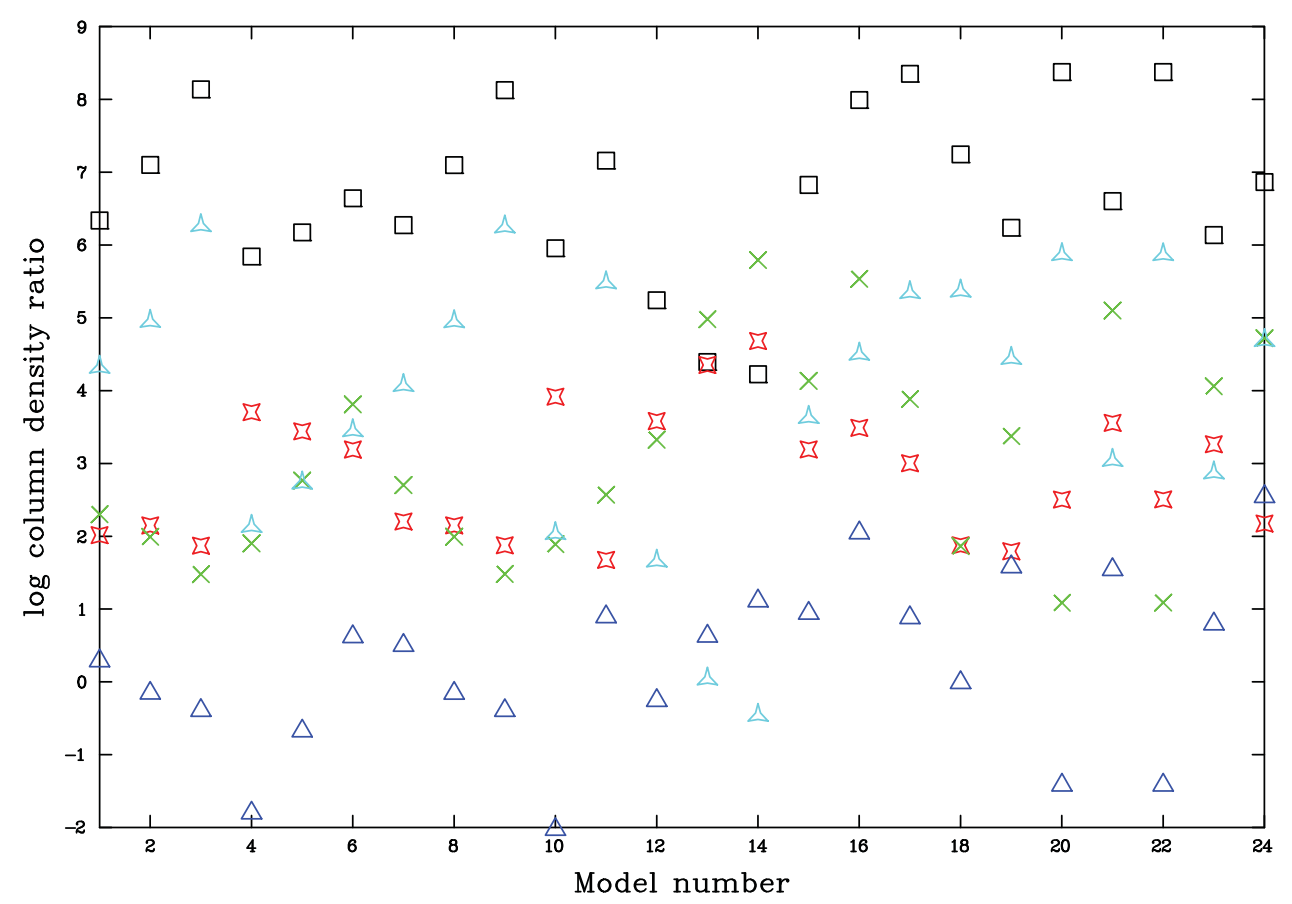

Figure 4. Theoretical fractional abundances ratios for the grid of UCL_CHEM models at high $\mathrm{A}_{V}(10 \mathrm{mags})$. The black squares are $\mathrm{CO} / \mathrm{HCO}^{+}$ratios; red stars $=\mathrm{CO} / \mathrm{HCN}$; green crosses $=$ $\mathrm{CO} / \mathrm{CS}$; cyan triangles $=\mathrm{HCN} / \mathrm{CS}$; blue triangles $=\mathrm{HCN} / \mathrm{HCO}^{+}$. The numbering in the $\mathrm{x}$-axis refers to the Model number (see Table 1).

One notes first of all that the y-axis shows logarithmic values of column density ratios, and yet a very large range of ratios are displayed. None of them are anomalous in that the grid of models performed covers reasonable values of densities, cosmic ray ionozation rate, temperatures and radiation field. Viti et al. (2014) found that each subregion of the CND could in fact be fitted by more than one set of column densities and, more importantly, that there is not one single model that can explain all ratios: multiple gas components even within each sub region are required. In fact, the multi-analyses performed in Viti et al. (2014) seem to indicate that there has to be a pronounced chemical differentiation across the CND and that each subregion could be characterized by a 3-phase component ISM, with two gas phases at different densities but both subjected to a high ionization rate, and one gas component comprising of shocked gas where most likely the CS arises from.

\section{Concluding remarks}

Recent years have witnessed a plethora of extragalactic molecular surveys which brought detailed information about the amount and distribution of the molecular gas in different types of galaxies. High resolution multi-species as well as multi-line molecular observations coupled with multiple independent analyses take us a step closer towards disentangling the gas components and energetics of nearby galaxies.

\section{References}

Aladro, R., Martín, S., Martín-Pintado, J., et al. 2011, Aछ A, 535, A84 
Aladro, R., Viti, S., Bayet, E., et al. 2013, A\&A, 549, 39

Bolatto, A., Wolfire, M., \& Leroy, A. K. 2013, ARA\&A, 51, 207

Bell, T. A., Roueff, E., Viti, S., \& Williams, D. A. 2006, MNRAS, 371, 1865

Bisbas, T. G., Bell, T. A., Viti, S., Yates, J., \& Barlow, M. J. 2012, MNRAS, 427, 2100

Costagliola, F., Sakamoto, K., Muller, S., et al. 2015, A\&SA, accepted (arXiv:1506.09027)

García-Burillo, S., Combes, F., Usero, A., et al. 2014, A\& $A, 567,125$

Hailey-Dunsheath, S., Sturm, E., \& Fischer, J. 2012, ApJ, 755, 57

Israel, F. P. 2009, A\& A, 493, 525

Kamenetzky, J., Glenn, J., \& Maloney, P. R. 2011, ApJ, 731, 83

Kelly, G., Viti, S., Bayet, E., Aladro, R., \& Yates, J. 2015, A\&A, 578, 70

Krips, M., Martín, S., Eckart, A., et al. 2011, ApJ, 736, 37

Martín, S., Mauersberger, R., Martín-Pintado, J., Henkel, C., \& García-Burillo, S. 2006, ApJS, 164,450

Usero, A., García-Burillo, S., Fuente, A., Martín-Pintado, J., \& Rodríguez-Fernández, N. J. 2004, A\& $A, 419,897$

Van der Tak, F. F. S., Black, J. H., Schöier, F. L., Jansen, D. J., \& van Dishoeck, E. F. 2007, $A \mathscr{E} A, 468,627$

Viti, S., Collings, M. P., Dever, J. W., McCoustra, M. R. S., \& Williams, D. A. 2004, MNRAS, 354,1141

Viti, S., Jimenez-Serra, I., Yates, J. A., et al. 2011, ApJ, 740, 3L

Viti, S., García-Burillo, S., Fuente, A., et al. 2014, A\&A, 570, 28

Williams, D. A. \& Viti, S. 2013, Cambridge University Press 